\title{
Practice and Effect Evaluation of Drug Reorganization in Patients with Chronic Obstructive Pulmonary Disease
}

\author{
Peng Yan*, Laiji Zhou, Chunwang Hua, Ping Gan \\ Taizhou Second People's Hospital, Taizhou 225500, China \\ *Corresponding author: Peng Yan, 552052990@qq.com
}

\begin{abstract}
Objective: To explore the feasibility of drug reorganization in clinical practice through the drug reorganization service of clinical pharmacists for patients with chronic obstructive pulmonary disease (COPD) in respiratory and critical care department, and to evaluate the effect of the service in multiple dimensions. Methods: a total of 100 patients with COPD admitted from January to July 2021 were randomly divided into two groups with 50 patients in each group. One group was treated with drug reforming intervention and the other group was not treated with intervention. Statistics and data analysis were carried out from three aspects: patient compliance, satisfaction and medication deviation. Results: (1) compliance: $48 \mathrm{~h}$ after admission, there was no significant difference between the two groups $(\mathrm{P}>0.05)$; According to the data statistics on the day of discharge and after one month, the compliance of the two groups was statistically significant $(\mathrm{P}<0.05)$, which was higher in the reorganization group. (2) Satisfaction: There was no significant difference between the two groups after admission. However, after drug reforming intervention by pharmaceutical staff, the results of reforming group were better and statistically significant $(\mathrm{P}<0.05)$. (3) Medication deviation: There were 50 deviations in the reorganization group, of which drug omission accounted for $52 \%$, which was the main factor. After communicating with clinicians, the clinicians' acceptance rate of the reorganization scheme reached $94.12 \%$. Conclusion: The implementation of drug reorganization service can effectively prevent the medication deviation of COPD patients, save the cost of patients, improve the satisfaction and compliance, and ensure the medication safety of patients.
\end{abstract}

Keywords: Drug reforming; Compliance; Satisfaction; Medication deviation

Publication date: September 2021; Online publication: September 30, 2021

\section{Introduction}

Chronic obstructive pulmonary disease (COPD) is one of respiratory diseases, incidence rate is higher, especially in elderly patients. The patients are relatively old and have poor physical function ${ }^{[1]}$. They often merge many diseases, and patients have many kinds of medication. There are often drug deviations, that is, the difference between the dosage, variety, dosage form, taking method and cycle of medication. Therefore, the rationality and standardization of medication for such patients are particularly important ${ }^{[2]}$. Drug reorganization has been widely carried out in European and American countries. It is to collect and record the medication history of patients and form the medication list before admission ${ }^{[3]}$. Pharmaceutical personnel, doctors, nurses and other parties work together to accurately intervene unreasonable medication behaviors by comparing with the medication list before admission, so as to ensure the safety and rationality of medication ${ }^{[4]}$. At present, the work has been incorporated into the daily assessment of pharmacists in developed regions abroad, but our work is still in the development stage, and there is no unified standard for system and mode ${ }^{[5]}$. This study carries out drug reorganization for COPD patients, collects relevant data 
and makes statistical analysis, draws relevant conclusions according to the results, and deeply discusses the necessity and importance of drug reorganization, so as to provide reference for the establishment of drug reorganization mode for COPD patients.

\section{Data and methods}

\subsection{Subjects}

100 patients with COPD admitted to our hospital from January to July 2021 were randomly selected and divided into two groups, with 50 people in each group. The subjects in the reorganization group carried out drug reorganization services within one day after admission or admission.

\subsection{Methods}

(1) COPD patients were screened out through the admission diagnosis of medical record system; (2) Record the medication list of the patient by asking about the medication of the patient. (3) According to the medication situation, drug reorganization is carried out, including stopping taking the drugs before admission, continuing to take the drugs before admission, adding new drugs, and forming a new drug list [6].

\subsection{Evaluation criteria}

\subsubsection{Compliance}

valuate the compliance of patients in three time periods, and fill in the compliance evaluation form, which is $48 \mathrm{~h}$ after admission, the day of discharge and one month after discharge. The evaluation mainly includes four parts: (1) whether to take medicine continuously (2) whether to forget to take medicine (3) whether to stop taking medicine when symptoms improve (4) whether to take the dose as required, 1-4 points for each part. After statistics, the scores in the scoring table are summarized. If the score is $\geq 12$, the compliance is good, and if the score is $<12$, the compliance is poor.

\subsubsection{Satisfaction}

Survey shall be conducted on the day of discharge. The evaluation includes six parts (1) service attitude (2) effect of diagnosis and treatment (3) diagnosis and treatment scheme (4) drug cost (5) medication guidance (6) rehabilitation guidance. Each part has 1-4 points. After statistics, the scores are summarized. If the score is $\geq 18$, the satisfaction is good, and if the score is $<18$, the satisfaction is poor.

\subsubsection{Medication deviation}

Register the medication situation of patients, record the medication deviation such as repeated medication, medication without indication and drug omission, and summarize the data for analysis.

\subsection{Statistical Analysis}

SPSS 22.0 software was used for data analysis, and the measurement data were tested by $\mathrm{T}$ test and expressed as $(\mathrm{x} \pm \mathrm{s})$. Statistical data were compared by $\chi^{2}$ test, $\mathrm{P}<0.05$, indicating statistical significance.

\section{Results}

\subsection{Compliance}

patients' compliance was investigated and evaluated in three different time periods, which were $48 \mathrm{~h}$ after admission, the day of discharge and one month after discharge. The results were expressed by the good compliance rate (good compliance rate $=$ number of people with good compliance $/$ total number). After statistics, the results of good compliance rate are as follows: (1) 48h comparison between the two groups, 
( $\mathrm{P}>0.05)$; (2) Comparison between the two groups on the day of discharge $(\mathrm{P}<0.05)$; (3) comparison between the two groups one month after discharge $(\mathrm{P}<0.05)$. See Table 1. After pharmaceutical care, the good compliance rate on the day of discharge and one month after discharge was significantly higher than that of 48 hours after admission $(\mathrm{P}<0.05)$. See Table 2. and Table 3.

Table 1. Comparison of good compliance rate between the two groups (\%)

\begin{tabular}{cccc}
\hline Group & 48h after admission & Discharge day & 1 month after discharge \\
\hline Non reforming group $(\mathrm{n}=50)$ & 52 & 58 & 44 \\
Reforming group $(\mathrm{n}=50)$ & 54 & 82 & 78 \\
$\mathrm{X}^{2}$ & 0.04 & 6.857 & 12.148 \\
$\mathrm{P}$ & 0.841 & 0.009 & 0.000 \\
\hline
\end{tabular}

Table 2. Comparison of good compliance evaluation rate of reorganization group at $48 \mathrm{~h}$ after admission and on the day of discharge $(\%)$

\begin{tabular}{cc}
\hline Time & Good rate \\
\hline 48h after admission & 54 \\
Discharge day & 82 \\
$\mathrm{X}^{2}$ & 9.007 \\
$\mathrm{P}$ & 0.003 \\
\hline
\end{tabular}

Table 3. Comparison of good compliance evaluation rate of reorganization group at $48 \mathrm{~h}$ after admission and 1 month after discharge $(\%)$

\section{Time}

48h after admission

One month after discharge

$$
\mathrm{X}^{2}
$$$$
\mathrm{P}
$$

\section{Good rate}

54

78

6.417

0.011

\subsection{Satisfaction}

comparison the satisfaction survey was conducted when the patients were discharged from the hospital. The recovery rate of the questionnaire in both groups was $100 \%$. A total of 1 person in the reforming group and 7 persons in the non-reforming group are dissatisfied. After statistical analysis, $\mathrm{P}<0.05$, there was statistical significance between the two groups. See Table 4.

Table 4. Comparison of patient satisfaction

\begin{tabular}{ccc}
\hline Group & Satisfied (example) & Dissatisfied (example) \\
\hline Non reforming group $(\mathrm{n}=50)$ & 43 & 7 \\
Reforming group $(\mathrm{n}=50)$ & 49 & 1 \\
\hline $\mathrm{X}^{2}$ & & 4.891 \\
$\mathrm{P}$ & 0.027 \\
\hline
\end{tabular}




\subsection{Analysis on medication deviation of reforming group}

\subsubsection{General data and types of medication deviation}

In the study of 50 patients in the reorganization group, the types of drugs used per capita reached $(6.96 \pm$ 2.03), involving 425 medical orders, including 17 patients with medication deviation and 127 medical orders, including 50 with deviation, including drug omission, repeated medication, frequency error, etc. See Table 5.

Table 5. Types of medication deviation

\begin{tabular}{ccc}
\hline Type & Number of articles & Percentage (\%) \\
\hline Drug omission & 26 & 52 \\
Repeated medication & 7 & 14 \\
Frequency error & 6 & 12 \\
Medication without indication & 4 & 8 \\
Dose error & 4 & 8 \\
Incompatibility or adverse interaction & 3 & 6 \\
\hline
\end{tabular}

\subsubsection{Adjustment of medication deviation}

According to medication deviation, the clinical pharmacist shall reorganize the scheme and feed it back to the attending physician. In this study, pharmacists intervened in 50 doctor's orders, involving 17 patients, including 25 suspension of medication, 12 adjustment of medication and 28 adjustment schemes; Results only one patient's reorganization scheme was not accepted, and the acceptance rate was $94.12 \%$.

\section{Discussion}

With the gradual advancement of pharmaceutical reform in China, drug reorganization has been paid more and more attention with the attention of clinical pharmacists and pharmaceutical care. The author focuses on evaluating and analyzing the effect of drug reorganization in COPD patients in several different dimensions, as follows:

\subsection{Effects of drug restructuring services on medication compliance}

Medication compliance reflects the degree of cooperation of patients with clinical treatment and plays an important role in the process of patient treatment. The results showed that the compliance of the reformed group was better than that of the non-reformed group on the day of discharge and one month after discharge, and the compliance of the two groups on the day of discharge and one month after discharge was better than that at admission. The patients in the study are older, have decreased physical function and memory, and are easy to forget to take medicine. Clinical pharmacists should pay more attention to this kind of population in their daily work. Therefore, such people need regular education to improve the medication compliance of such patients.

\subsection{Impact of drug reorganization on satisfaction}

Satisfaction is a response of patients to their actual feelings about the clinical treatment process. After research, the satisfaction of the two groups has been significantly improved after pharmaceutical care, which also shows that the clinical service of pharmaceutical personnel can improve the satisfaction of patients to a certain extent. 


\subsection{Intervention of clinical pharmacists on medication deviation}

The intervention of medication deviation is an important link in drug reorganization. In this study, most patients are older and have basic diseases. There are 425 medical orders involving drugs in the study, including 50 medical orders with medication deviation, and the incidence of medication deviation is $11.8 \%$. This may be related to the behavior of patients in the process of clinical treatment, including voluntary withdrawal and unauthorized change of drugs. At the same time, the treatment level and sense of responsibility of the attending doctors are also different. Unreasonable treatment and non-standard medication may also be part of the reasons for medication deviation.

To sum up, COPD is one of the common diseases in the respiratory and critical care department. The patients are older, have weak memory, low compliance, and medication deviation is common. Drug restructuring services can not only improve the above situation, but also ensure the medication safety of COPD patients. However, the development of drug reorganization still has a long way to go. It needs not only pharmaceutical personnel, but also the cooperation of doctors and nurses. The establishment of the reorganization scheme can help reduce the medication deviation, patients can better follow the doctor's advice, improve the treatment effect and improve the patient satisfaction. At present, the acceptance rate of drug reorganization in China is lower than that in developed countries, and it is still in the exploratory stage. It is not paid enough attention and has not been widely used. However, this work is the key development direction of pharmaceutical work in the future and needs to be gradually popularized. Before popularization, we should do a good job in paving the way. First, we should train medical personnel and pharmacists in relevant knowledge. Let them understand the purpose and significance of drug reorganization, increase the communication and cooperation among doctors, drugs and nurses, and better promote the process of drug reorganization; Secondly, it is necessary to improve the position of clinical pharmacists in the process of drug reorganization. The division of labor and responsibilities of doctors, drugs and nurses in the process of clinical treatment are different. Doctors pay more attention to the diagnosis and treatment of diseases. Nurses are mainly responsible for specific operation and daily nursing. Pharmacists have rich knowledge of pharmacology and pharmacy and are more professional in the use of drugs. In recent years, drug reorganization has been the daily work of clinical pharmacists in many countries, and it is an important guarantee measure for drug safety; At the same time, we should establish a standardized and standardized drug reorganization system to promote in the whole hospital, integrate drug reorganization into the medical record system by using information means, and gradually improve the hospital network service framework.

\section{Funding}

2021 clinical medical science and technology development fund of Jiangsu University (Jiangsu University Medical Department [2021] No. 8), Project No.: JLY2021189.

\section{Disclosure statement}

The author declares no conflict of interest.

\section{References}

[1] Xie Y, Liu C, Zhuang H, et al., 2021, Discussion on the Medication law of Liu Xiaohong in the Treatment of Chronic Obstructive Pulmonary Disease. Journal of Guangzhou University of Traditional Chinese Medicine, 38 (4): 810-815. Doi: 10.13359/j.cnki.gzxbtcm. 021.04.029

[2] He H, Guo S, Song Y, et al., 2020, Study on the Medication Law of Professor Sun Zengtao in the Treatment of Chronic Obstructive Pulmonary Disease based on Data Mining. Chinese Traditional Medicine Emergency, 29 (4): 604-607. Doi: 10.3969/j.issn.1004-745X.2020.04.011 
[3] Yang Y, Li G, Zhang Z, et al., 2021, Practice of Pharmaceutical Intervention by Clinical Pharmacists in Respiratory Medicine. Modern Medicine and Health, 37(9): 1582-1585. Doi: 10.3969/j.issn.1009-5519.2021.09.045

[4] Zhao F, Qin Y, Zhang X, et al., 2021, Analysis of Inpatient Drug Reorganization in Gastroenterology Department. Chinese Journal of Hospital Pharmacy, 41(11): 1142-1145. Doi: 10.13286/j.10015213.2021.11.11

[5] Zhao S, Fan P, Cai H, et al., 2021, Experience of Clinical Pharmacists in Drug Reorganization in the Practice of Antithrombotic Drug Treatment. Henan Medical Research, 30(18): 3371-3374. Doi: 10.3969/j.issn.1004-437x.2021.18.032

[6] Wu W, Zhang R, Liu H, et al., 2018, Implementation and Clinical Evaluation of Whole Process Pharmaceutical Care for Patients with Chronic Obstructive Pulmonary Disease. China Medicine and Clinic, 18(5):718-720. Doi: 10.11655/zgywylc2018.05.019 